\title{
Online Collaborative Decision Making System for Pavement Maintenance
}

\author{
Ting Peng ${ }^{1,}$, Zhihang Liü, b, Lei Guo ${ }^{1, ~ c}$, Xiaoling Wang 2, d \\ ${ }^{1}$ MOE Special Region Highway Engineering Lab, Chang'an University, Xi'an, 710049 China. \\ ${ }^{2} X i ' a n$ Branch of the People`s Bank of China, Xi'an, 710004 China \\ at.peng@ieee.org, b2412352858@qq.com, c 752464906@qq.com, d1278766827@qq.com
}

Keywords: pavement maintenance; decision making system; OWA operator theory; the support degree.

\begin{abstract}
Nowadays, there are some shortcomings of road maintenance decision-making system for pavement maintenance, which only depends on the expert's experience and the improper maintenance time. Therefore, this paper introduces the on-line collaborative decision-making system of pavement maintenance. The system is based on the fully using of expert experience, and combines the efficient processing speed of the computer. Therefore, this paper can provide a scientific method for selection of optimal maintenance decision and optimal maintenance time. Considering the type of different pavement structure, environmental factors, traffic factors, a large number of test data and the prediction of pavement performance, based on the using of computer technology, this paper achieves the purpose of the optimal decision of maintenance plan and maintenance time, and on this basis ,constructing the evaluation system of decision consistency. This method improves the correctness of maintenance decision making, and makes the decision more scientific and economical.
\end{abstract}

\section{Introduction}

With the continuous improvement of the construction of infrastructure, the traffic mileage of highway is increasing rapidly, the occupancy rate of large car and the phenomenon of overloading has increased dramatically. Now, the highway in china has entered the maintenance period. The decision making system of Highway maintenance has appeared a single expert decision-making, pavement management system, maintenance expert system, and other forms. Single expert decisionmaking relies mainly on the experience of a single expert to develop a maintenance plan. Pavement management system gives the final pavement maintenance program through analyzing the basic information provided by the department of Pavement management. Expert system utilizing the experience of experts in the system, customizes database management module, pavement performance evaluation module, pavement performance prediction module, economic analysis and program decision making module, then making maintenance decision [1]. And finally realize the automation of the decision-making process. However, there are many shortcomings in the road maintenance decision making method above: in a single expert decision making system, the decision making mainly depending on the individual, so, the subjectivity is too strong. The decision of pavement maintain made by the pavement management system is influenced by the accuracy of data.; The process of decision-making is based on the mechanism of the expert system to achieve, so people's subjective initiative is difficult to reflect.

Therefore, based on the original maintenance decision-making method, combined with expert database experts' experience and the method of decision making, realizing the combination of the mechanism reasoning and the person's subjective initiative, A multi-objective group on-line collaborative decision-making method is constructed, which can determine the optimal maintenance scheme and maintenance timing of the road. Multi objective decision making method is based on the principle of multiple objectives to determine the pros and cons of the decision-making process. In the pavement maintenance decision-making, the key problem to be solved is how to find the best maintenance strategy and the implementation time of the conservation strategy under the condition 
that the budget funds and other resources are certain, so as to realize the maximization of economic benefit so as to realize the maximization of economic benefit. In other words, under the premise of meeting the pavement performance, to minimize the pavement maintenance cost. In order to solve this problem, this paper proposes a method based on multi objective group online collaborative decision making.

\section{Feasibility Study}

\subsection{Preferred Maintenance Programs.}

The main task of pavement maintenance decision-making is to obtain the performance prediction curve which is consistent with the actual situation. The performance prediction curve is obtained by fitting the actual road performance data at any time, which mainly relies on the attenuation mechanism of different types of structure, environmental factors and traffic factors. The performance prediction curve can be used to query the database of the road maintenance strategy and obtain all the available maintenance strategies. By road maintenance strategy set and performance prediction curve, you can get all the pavement maintenance measures and the implementation of the combination of time. In all of the pavement maintenance scheme, some schemes have good decision-making methods, but the cost is high; some schemes have low maintenance cost, but the maintain method is not suitable for the pavement diseases [2].Therefore, it is necessary to optimize the combination of all the pavement maintenance measures and the implementation time points, in order to obtain the best maintainable plan. By evaluating the technical and economic indicators of the combinations to calculate the various combinations of the required materials, equipment, manpower, capital, road performance indicators such as the rate of increase. At the same time, the maintain cost and performance improvement of different maintain measures under different performance conditions and at different times were calculated. Through various departments' online collaboration, listen to the views of engineers and other steps , on the basis of the principle of taking the preventive maintenance and reducing large and medium repair, obtain the maintain program, which can meet the requirements of pavement life cycle and has the lowest cost[3].

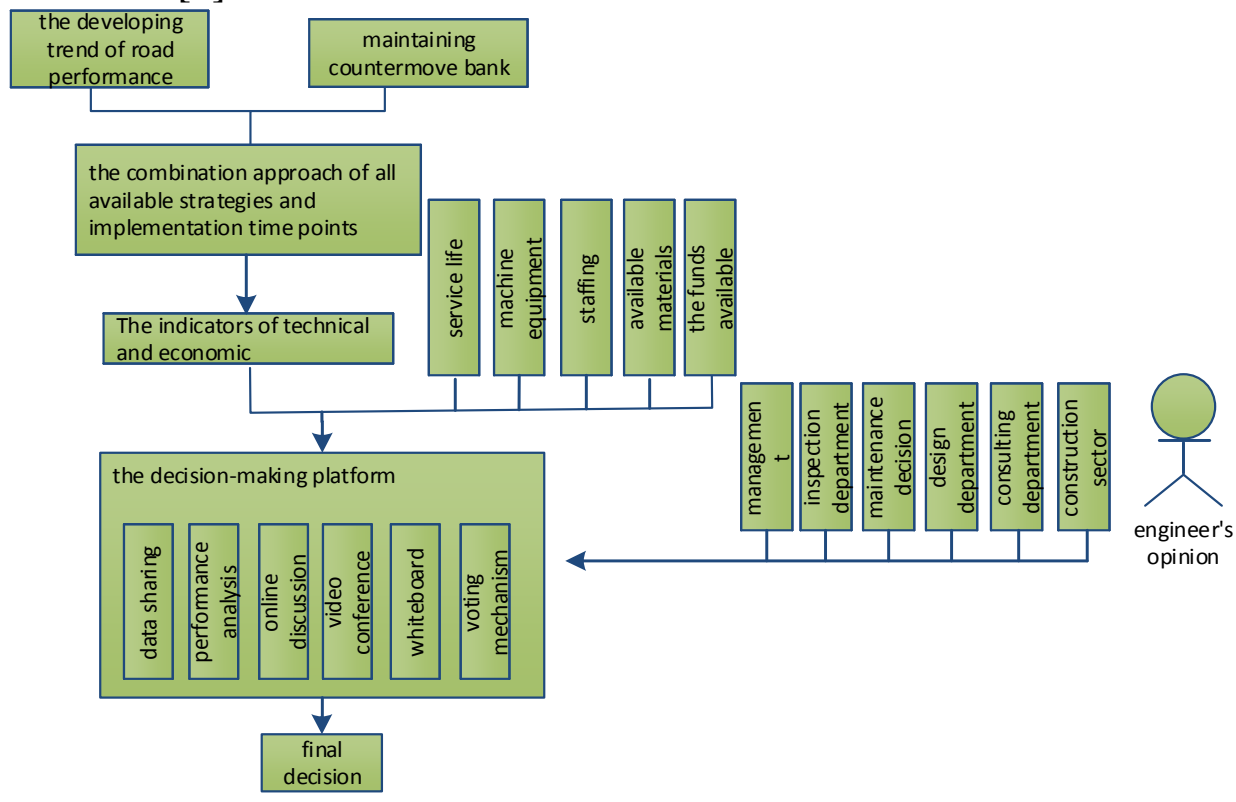

Fig. 1 the process of preferred method

Assuming that a certain road appears disease, need to maintain, through the road performance prediction curve and conservation strategy database, we can get a total of $n$ conservation strategies and implementation time of the combination of programs. These maintain programs are denoted as $A_{i}, A_{i}=\left(t_{i}, w_{i}\right), i=1,2,3 \ldots n$, among which $t_{i}$ stands for the timing of maintenance and $w_{i}$ represents the craft used in the maintenance and maintenance. For any plan $A_{i}$, The costs, manpower, materials and equipment needed for maintenance are denoted as $C F_{i}, C P i, C M i$ and $C T_{i}$ respectively.After the maintenance program is expected to improve the expected traffic recorded as $P P_{i}$. 
The necessary and sufficient conditions for the feasibility of the combined scheme are that the available resources must be greater than or equal to the required resources, as shown in the formula (1).

$$
C F_{i} \leq A V(C F) \cap C P_{i} \leq A V(C P) \cap C M_{i} \leq A V(C M) \cap C T_{i} \leq A V(C T)=\text { True }
$$

In the formula:

$A V(x)$-represents the amount of $x$-class resources in the available resources;

If the conditions are not satisfy this formula, it is considered that the scheme does not have the conditions for implementation and the scheme is directly removed from the composition scheme in the actual decision.

When the conservation program are over totally evaluated, the total cost can be written as a function of resource consumption, as shown in the formula (2).

$\operatorname{Cost}\left(A_{i}\right)=f\left(C F_{i}, C P_{i}, C M_{i}, C T_{i}\right)$

Among them, the detailed expression of the function $f$ is decided according to the practical experience and the concrete circumstance of the maintenance decision maker. However, different decision makers believe that each parameter has a different weight in this function. To solve this problem, the default cost weight is 0.85 , and the other weight is 0.15 . After the implementation of the combined program $A_{i}$, the economic benefits generated during the term of use are judged as the standard of tolls and the extension of the service life. Tolls are mainly affected by various types of vehicles, vehicle mileage, so the difference between the programs is very small, which can be ignored in the evaluation process. Consequently, economic benefit indicators can be expressed as service life $T\left(A_{i}\right)$. The final evaluation of the maintain program is written in the formula (3).

$$
\operatorname{Eval}\left(A_{i}\right)=g\left(\operatorname{Cost}\left(A_{i}\right), T\left(A_{i}\right)\right)=S V\left(A_{i}\right)(P / F, i, 20)-\operatorname{Cos} t\left(A_{i}\right)\left[1+\sum_{j=1}^{n}\left(P / F, i, j T\left(A_{i}\right)\right)\right]
$$

$S V\left(A_{i}\right)$---- After the conservation program was implemented 20 years, the pavement performance residual value are expressed with current value,which are equaled as $\frac{20 \% T\left(A_{i}\right)}{T\left(A_{i}\right)} \times \operatorname{Cost}\left(A_{i}\right)$,\% is the remainder sign;

$(P / F, i, j)$--- The discount rate $i, j$-the $j$-th year discount factor, herein take $i=4 \%$;

$n$--- The number of the service life cycle of maintain plans included in the analysis period, $n=20 \div T\left(A_{i}\right)$;

In the evaluation of the pavement maintenance program, the net annual value is linearly related to the amount of road condition improvement and the increase in traffic volume in the analysis period (the asphalt pavement of highway take 20 years in analysis period) [4], among which, the amount of road condition improvement is reflected in the road performance boost and the extension of road service life. The overall assessment of the conservation program is defined as a function g, which is a function of the cost of maintenance and the benefits of generation, where the expected costs and benefits are calculated at present value. Through this method can determine technics and economic indicators of the maintain programs, so as to determine the available programs and eliminate the programs which does not meet the requirements [5]. If you want to get the maintain programs, which meet the performance requirements of the road surface life cycle and the lowest cost, you must get all of the conservation measures and the implementation of the time point of the combination program, and further selection

\subsection{Selected Conservation Combination Programs.}

Previously, the selection of conservation combination program is mainly relying on expert jury, but the selection of the jury expert mostly relies on conservation practice experience, each person's practical experience is different and the program information they got is different ,which resulted in the scoring deviation of each maintain programs. On the other hand, the summary results of the program scoring can't accurately reflect the views of all experts. To solve this problem, an on-line collaborative maintenance decision-making method is proposed and applied in this paper.

On-line collaborative maintenance decision-making platform is the system of decision-making, that is, in the course of road maintenance decision-making management, carrying out mechanism analysis on technical management and determining the interaction with the work-related personnel and data flow, in order to determine the final maintenance decision-making. The platform is mainly 
composed of data sharing, teleconferencing, whiteboard, workflow and decision-making voting mechanism, which provide efficient and convenient services for road operations management, road detection, maintenance decision-making and construction departments. The workflow of platform is shown in Fig. 2.
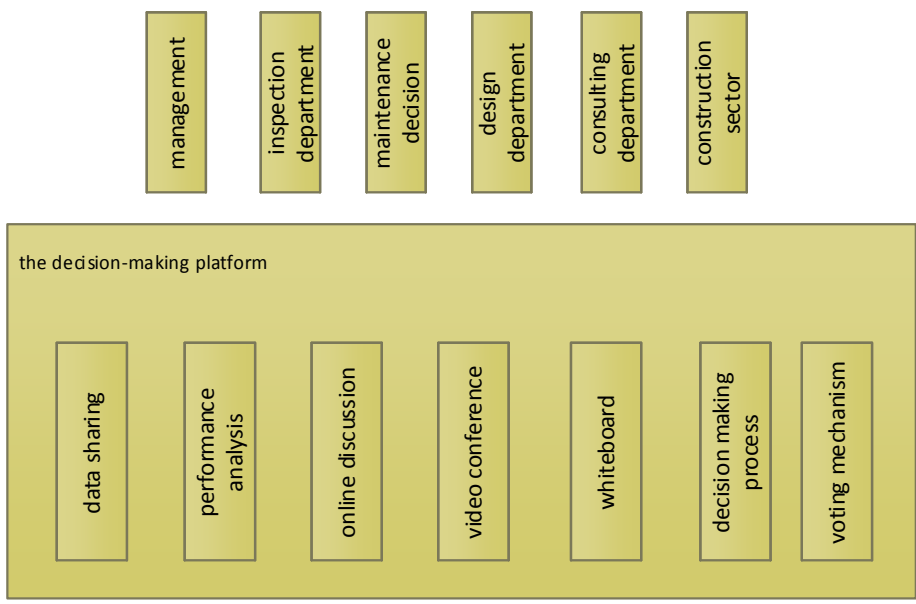

Fig. 2 the workflow of platform

Through the collaborative decision-making platform, the decision-makers from various departments combined with road maintenance engineers' analysis results, according to the road performance, disease mechanism, disease development trend, suitable maintenance measures and time points, maintenance cost as well as expected effect for further and whole indicators' analysis. And then basing on the group solution rules, the decision - making of multi - decision - makers on the final maintenance plan is realized.

Assuming that a certain road appears disease, need to maintain, through the road performance prediction curve and conservation strategy database, we can get a total of $n$ conservation strategies and implementation time of the combination of programs. These maintain programs are denoted as $A_{i}, A_{i}=\left(t_{i}, w_{i}\right), i=1,2,3 \ldots n$, with m indicators, we can evaluate the combination programs of the $\mathrm{n}$ kinds of maintenance strategy and the implementation time points, each indicator is denoted as $C_{j}, j=1$, $2, \ldots, m . Q$ decision makers from multiple departments are denoted as $B k, k=1,2, \ldots, q$. scoring for the indicators of each maintenance program respectively, the result is remarked as $Z_{i j}$, and the range of the decision-maker's score is $[0,1]$, the weights of each indicator is remarked as $\omega_{j}, j=1,2 \ldots m$.

In order to solve the problem that the decision-makers have different opinions on the weight, the $O W A$ operator theory is proposed and used for the first time. The $O W A$ operator theory is also called location weighting theory. It is merely related to location and has nothing to do with other factors. The evaluation index values are arranged according to a certain order, and the final score is obtained by orderly weighted average of the conservation plans needing to be evaluated. The empirical results show that the position weight data are usually concentrated in the middle position and gradually decrease in both sides. The more the data are, the more obvious the rule is. By the data fitting, The $O W A$ operator can fit the law of the normal distribution probability density function very well. (The details are shown in Fig. 3)

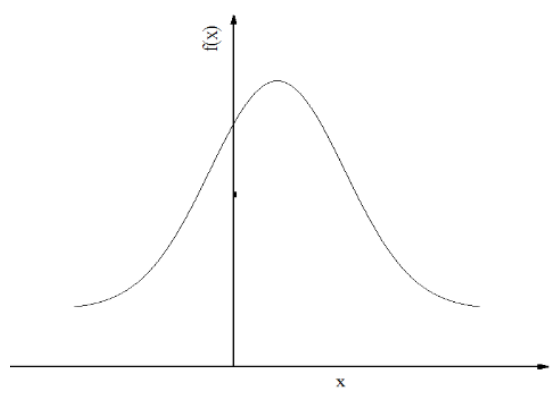

Fig. 3 the normal distribution probability density function

Assuming that the position weight is a normal distribution, the design formula is shown as follows: 


$$
\omega_{j}=\frac{1}{\sigma \sqrt{2 \pi}} e^{-\frac{(j-\mu)^{2}}{2 \sigma^{2}}} \quad j=1,2 \cdots \mathrm{m}
$$

Initial assignment of position sequence $W=\left(\omega_{1}, \omega_{2}, \cdots \omega_{\mathrm{m}}\right)=(1,2 \cdots m)$, then

$$
\begin{aligned}
\mu & =\frac{1+2+\cdots+m}{m}=\frac{m+1}{2} \\
\sigma^{2} & =\frac{(1-\mu)^{2}+(2-\mu)^{2}+\cdots+(m-\mu)^{2}}{m}=\frac{(m+1)(2 m+1)}{24}
\end{aligned}
$$

In the case of expectation and variance known, Substituting Equation (4) to find the expression of $\omega_{j}$. By further sorting out the position weight, we can get:

$$
\omega_{j}^{\prime}=\frac{\omega_{j}}{\sum_{j=1}^{m} \omega_{j}}
$$

Then, in the case of position weight sequence $W=\left(\omega_{1}, \omega_{2}, \cdots \omega_{m}\right)=(1,2 \cdots m)$ and the score sequence $Z_{i j}$ determined, the average score of a certain program is calculated as follows:

$$
O W A_{i}=\sum_{j=1}^{m} \omega_{j}^{\prime} z_{i j}
$$

In the formula:

$O W A_{i}$ - decisions on the weighted average of the indicators for the $i$-th conservation program.

$\omega_{j}^{\prime}$ - the position weight of the $j$-th index

$z_{i j}$ _ sorting the score of the $i$-th conservation programs evaluation in descending order, the score at position $j$;

Through the above steps solved the problem that the decision-makers have different opinions on the weight, the final score is calculated on the order-weighted average of the programs that need to be evaluated, which lays the foundation for the further evaluation of the program.

In this paper, regarding the weight of the support degree conversion coefficient as the degree of recognition of score of each expert on certain program, which solves the problem that the final decision can not accurately reflect the views of experts on conservation programs. Its' specific operation steps: Weight multiplied by the corresponding score, and then the sum of the product to get the final score of the program. All the opinions of experts was taken into account and captured in the final score. Mark the evaluation results of the first $\mathrm{k}$ decision maker on the first $i$ maintenance plan are as $P_{i}^{k}$, so $P_{i}^{k}=O W A_{i}$. The support degree of other decision makers in the group on the judgments of the $k$-th decision makers shown in the following formula:

$$
t_{k}=\sum_{d=1}^{q} \operatorname{Sup}\left(P_{i}^{k}, P_{i}^{d}\right)
$$

In the formula:

$t_{k}$ - The support degree of other decision makers in the group on the judgments of the $k$-th decision makers, which is a two valued function, and whose Value was shown in the formula (9).

$P_{i}^{k}$-The ordered weighted average of the decisions made by the $k$-th decision maker for the

$i$-th conservation program;

$$
\operatorname{Sup}\left(P_{i}^{k}, P_{i}^{d}\right)= \begin{cases}1, & \left|P_{i}^{k}-P_{i}^{d}\right|<\beta \\ 0, & \left|P_{i}^{k}-P_{i}^{d}\right| \geq \beta\end{cases}
$$

$\beta$ — The limit to judge whether the decision maker's opinion is consistent and the value is related to the range of $Z$. It can also be adjusted according to specific circumstances. In this study, the value is 0.02 . 
In this way, we get the $Q$ value from $Q$ decision maker $\left(P_{i}^{k}, t_{k}\right)$, among them: The weighting sequence $V=\left[v_{1}, v_{2}, \ldots, v_{q}\right]$ is calculated according to formula (10):

$$
v_{k}=\frac{Q\left(t_{k} / q\right)}{\sum_{k=1}^{q} Q\left(t_{k} / q\right)}
$$

Where $Q(x)$ is defined as below:

$$
Q(x)=\left\{\begin{array}{cc}
1, & x \geq 0.8 \\
2 x-0.6, & 0.3<x<0.8 \\
0, & x \leq 0.3
\end{array}\right.
$$

Then $Q(x)$ is a piecewise function, of which functional expression was shown in the Fig. 4

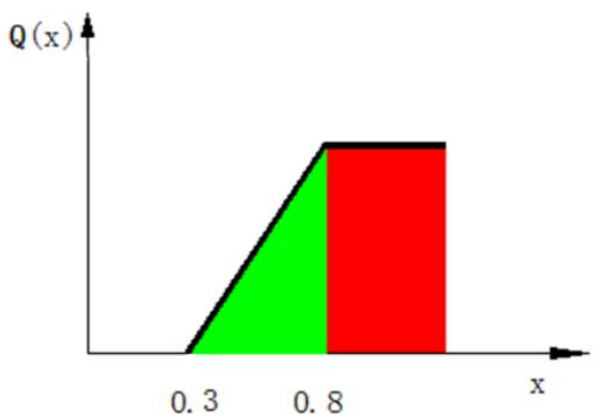

Fig .4 piecewise function of $Q(x)$

Final score of the conservation program:

$$
p_{i}^{\text {majority }}=\sum_{k=1}^{q} v_{k} P_{i}^{k}
$$

The final score for a given conservation plan is the result of the evaluation of the decision-maker in the decision-making group deal with the conservation plan based on the group solution rule. If the score of $p_{i}^{\text {majority }}$ is high, which indicates that the program is more recognized by most of the decisionmakers in the decision-making group. The feasibility of the program is relatively large.

\section{Decision - Making Program Detection}

The indicator $p_{i}^{\text {majority }}$ reflects the common view of all decision makers in the decision-making group on the conservation program .But it does not reflect the degree of consistency of the decisionmakers on the conservation program. In order to reflect the decision-makers on the degree of consistency of conservation programs, in this paper, the consistency of decision-making is used to measure that weather the results of each decision maker are consistent with the results of other decision makers. The degree of consistency of the decision is defined as [0,1].1 means that all decision-makers in the group have the same opinion. And 0 means that the decision-makers in the group failed to reach an agreement.

Marking $D$ as a team, which is made up of all decision makers. Among them, the $k$-th decision maker is recorded as $D_{k}(k=1,2, \ldots, q)$.First of all, each decision maker makes separate decisions on the issues involved, in this way, we can obtain an evaluation result made up of $Q$ separate maintenance plan, That is $S M_{1}, S M_{2}, \ldots, S M_{q}$. Among them $S M_{k}$ is a collection, which is made up of $\left(A_{i}\right.$, score $\left._{i}\right)$ ( $i=1,2, \ldots, \mathrm{n}$ ), $A_{i}$ represents the ith alternative , score $_{i}$ represents the score of the maintenance plan, which is a decision maker's judgment on all feasible alternatives.

In the decision making group, each decision maker forms an ordering set $R=\left[R_{1}^{k}, R_{2}^{k}, \ldots, R_{\mathrm{n}}^{k}\right]$ for all feasible schemes according to the score of the maintenance plan .Among them $R_{i}^{k}$ represents the 
$\mathrm{k}$-th decision maker's order on the ith alternative, Range of values is [0,n], maintenance plans with the same score have the same sort of results.

The sorting results for all the individual decision-makers in the group are calculated to obtain the group ordering of any one of the alternatives. Compare the ranking of alternatives with each decision maker and the sort of group, Calculating the distance between the decision maker and the team decision. As follows:

$$
D_{i}^{k}=\frac{\left|R_{i}^{g}-R_{i}^{k}\right|}{n-1}
$$

In the formula:

$D_{i}^{k}$ - The distance between the decision of the kth decision maker on the ith scheme and the decision of the group, $D_{i}^{k}$ is defined within the unit interval. $D_{i}^{k} \in[0,1]$

$n$ - The number of alternatives

$R_{i}^{g}$-The results of group decision making on the ith scheme, if $R_{i}^{g}=R_{i}^{k}, D_{i}^{k}=0$; If the difference between $R_{i}^{g}$ and $R_{i}^{k}$ reaches the maximum value $n-1, D_{i}^{k}=1$.

The degree of consistency of decision made by the q-decision makers in the decision-making group on the i-th scheme is calculated as follows:

$$
C D_{i}=1-\frac{\sum_{k=1}^{q} D_{i}^{k}}{q}
$$

The degree of consistency of decision made by the group is calculated by the formula:

$$
C M=(1-\beta) \frac{\sum_{i=1}^{n} C D_{i}}{n}+\beta C D_{\text {sol }}
$$

In the formula:

$C D_{\text {sol }}$ - The degree of consistency of decision program, which is the program that ranking first in team decision making.

$\beta$-defined in the unit interval, in this study, the value is 0.7

The calculation formula of the closeness of the decision of any one decision-making and group decision-making results is as follows:

$$
P M_{k}=(1-\beta) \frac{\sum_{i=1}^{n}\left(1-D_{i}^{k}\right)}{n}+\beta\left(1-D_{s o l}^{k}\right)
$$

In the formula:

$D_{\text {sol }}^{k}$ - The distance between the team decision results and the decision given by the K-th decision maker

If the consistency $C D_{i}$ of the decisions obtained from the i-th maintenance program is high, which means that the decision makers have a high consistency in the judgment of the scheme, the results obtained are more reliable, and the feasibility is higher; Instead, which shows that the decision makers have great differences in the scheme, and the credibility of the evaluation results is low relatively, and the feasibility is low.

\section{Conclusion}

The application of ordered weighted averaging operator in pavement maintenance decision is proposed in the first time, solved the problem that the weight of the evaluation index is different. In addition, the concept of distance is proposed to measure the support degree of each expert to the evaluation plan, the distance of ranking results is used to show the difference between the decision maker on the maintain program and the group decision making, which is convenient to make a 
qualitative comparison of maintenance decision making and provide a scientific method and basis for the final decision of road maintenance program.

\section{References}

[1]. Snaith M.S. Pavement Maintenance and Management System Manual [K]. The University of Birmingham UK. 1984

[2]. Yuli Pan. The principle of pavement management system [M]. Beijing: China Communications Press, 1998

[3]. Xiang Yu. Research on decision making optimization of highway pavement maintenance management system [D]. Chengdu: Southwest Jiao Tong University. 2005

[4]. Hongyan Jiang. Research on highway pavement maintenance management system [D]. Xi'an: Chang'an University. 2006

[5]. Wan Zheng. Highway asphalt pavement maintenance decision of multi target control [D]. Harbin: Northeast Forestry University. 2014 